\title{
Industrial Application of Fuzzy Systems: Adaptive Fuzzy \\ Control of Solder Paste Stencil Printing
}

\author{
A. LOTFI and M. HOWARTH \\ Manufacturing Automation Research Group, Department of Mechanical and \\ Manufacturing Engineering, The Nottingham Trent University, Burton Street, \\ Nottingham, NG1 4BU, England
}

\begin{abstract}
This paper presents an adaptive fuzzy control algorithm for the control of the solder paste stencil printing stage of surface mount printed circuit board assembly. The proposed method of automatic solder paste stencil printing consist of four blocks; fuzzy feature extraction, defect classification of paste deposits, adaptive fuzzy rule-based model identification and subsequently an optimal control action for the stencil printing process. Experimental results are presented to illustrate the capability of the algorithm.
\end{abstract}

$\overline{1}$ The authors would like to acknowledge the support of the EPSRC (Grant GR/K76559) for this work conducted under the Design and Integrated Production targeted program.

Preprint submitted to Elsevier Preprint $\quad 21$ July 1997 


\section{INTRODUCTION}

Surface mount technology (SMT) is the dominant methodology in the assembly of sophisticated electronic devices [3]. It involves bonding electronic packages to printed circuit boards (PCBs) via solder paste pads. The solder deposits are accurately placed on the PCB by stencil printing, however, faults in the printing process are a major source of board failure. There will be significant economic benefits from enhanced product yield, if the process is controlled more accurately.

The small amount of solder that attaches a surface mount component to the circuit, serves as both the electrical and mechanical means of connection. In the SMT process, the most critical step is the solder paste stencil printing stage. If the correct amount of paste is not applied at this stage, later process steps cannot correct the situation. The solder paste stencil printing process is complex and has a number of parameters that can influence the final quality of the solder paste deposit.

To produce better solder paste deposits and to automate the process of stencil printing, the algorithm presented in this paper is proposed. The block diagram for control of the solder paste stencil printing stage of SMT is illustrated in Figure 1. The proposed architecture consists of four principal components. They are: a) fuzzy feature extraction b) defects classification using the fuzzy c- mean algorithm c) adaptive model identification employing an adaptive 


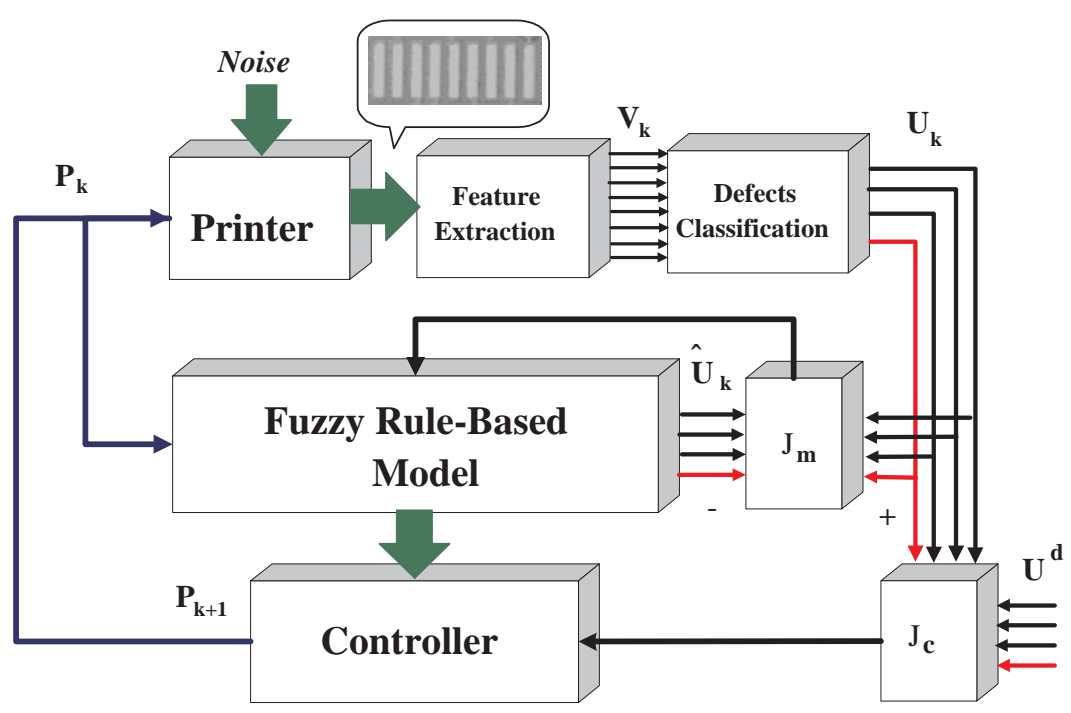

Fig. 1. Proposed block diagram of the automatic solder paste stencil printing control system.

fuzzy rule-based system and d) optimal controller. The following paragraphs describe the indicated elements of the proposed block diagram.

Applying a set of parameters $\mathbf{P}_{\mathbf{k}}$ to the printer produces a print which can be mapped to image space using a fast laser scanner. To reduce the dimensionality of the image space, each image will be transformed into a fuzzy feature vector $\mathbf{V}_{\mathbf{k}}$. A set of $m$ fuzzy geometrical features for each image is used to perform the conversion from range image data to feature vector $\mathrm{V}_{k}$. This procedure is considered as mapping from parameter space $\mathbf{P}_{\mathbf{k}}$ into feature vector $\mathbf{V}_{\mathbf{k}}$, i.e. $\mathbf{P}_{\mathrm{k}} \mapsto \mathbf{V}_{\mathrm{k}}$

To have an intuitive understanding of the above mapping, it is desirable to alter the feature vector $\mathbf{V}_{\mathbf{k}}$ into defect class membership functions (CMFs) $\mathbf{U}_{\mathbf{k}}$, using a classification method. We used the fuzzy c-mean algorithm to 


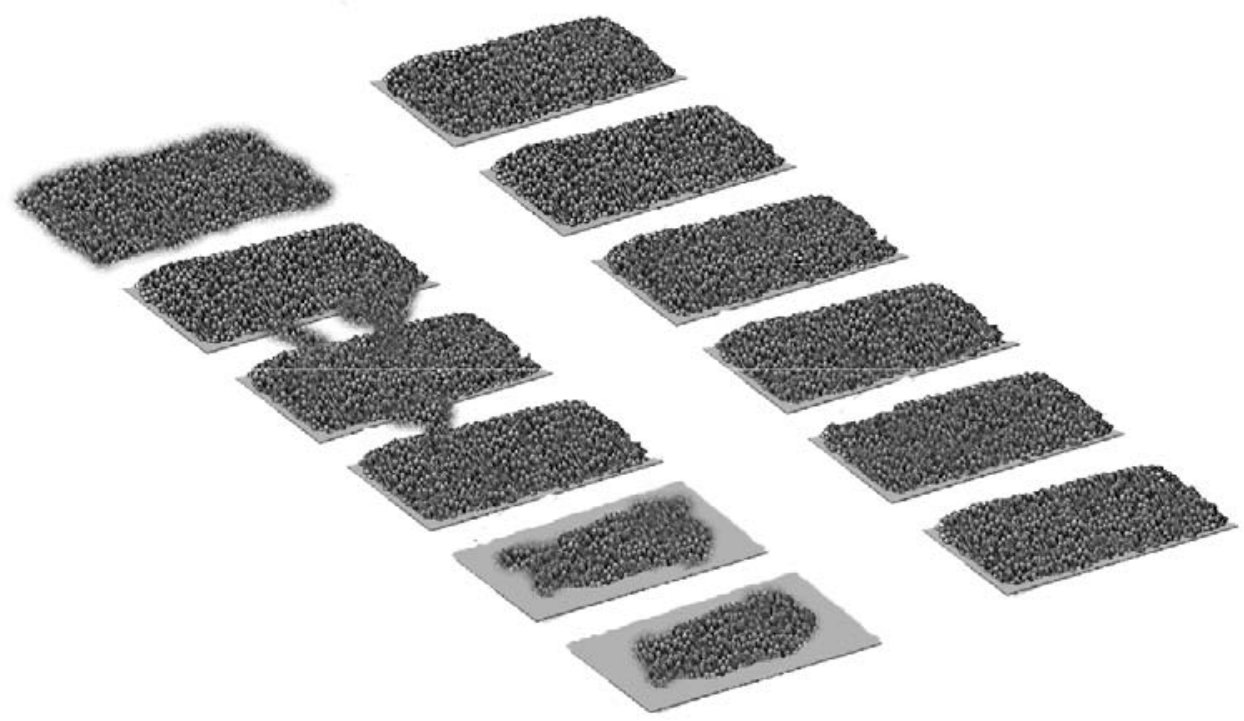

Fig. 2. 3-D image of solder paste deposits on a PCB representing typical defects. perform this transformation. The resulting output produces $q$ classes which ranged from ideal to bad where the CMF of each image represents the degree of membership into each class. Figure 2 illustrates a 3-D image of solder paste deposits on a PCB representing typical defects. The ideal shape of paste deposits is shown in the second row.

When direct mapping from $\mathbf{P}_{\mathbf{k}}$ to $\mathbf{U}_{\mathbf{k}}$, generated from two separate mappings i.e. $\mathbf{P}_{\mathbf{k}} \mapsto \mathbf{V}_{\mathbf{k}} \mapsto \mathbf{U}_{\mathbf{k}}$ is established, it enables us to create a model for the printing process using a fuzzy rule-based system (FRBS). FRBSs have demonstrated their capability for nonlinear function approximation. From the large set of experimentally obtained, input-output $\left(\mathbf{P}_{\mathbf{k}}, \mathbf{U}_{\mathbf{k}}\right)$ data, it is clear that the printing process produces a nonlinear response. Hence, a FRBS is ideally suited to modelling the nonlinear behavior of the stencil printing process.

To take into account the effect of unknown parameters and uncertainty in- 
volved in the printing process, the model identification will be accomplished employing an adaptive FRBS [7]. It will ensure that the error generated from the output of the model, $\hat{U}_{k}$, and CMF $U_{k}$ is minimized.

The requirement of the printer producing high quality output (paste deposit) can be translated into having a CMF with the highest value in the class of ideal deposit shape. Therefore the control action for each consequent print $\mathbf{P}_{k+1}$ will be in the form of settings that maximize the CMF for the ideal class based on the generated fuzzy model for the printer.

The presented work in this paper gives a brief introduction to the stencil printing stage of surface mount assembly process followed by a description of fuzzy feature extraction in Section 3. The defects classification method is explained in Section 4. The subsequent FRBS model and control strategy are described in Sections 5 and 6 respectively. The experimental results are presented in Section 7. Concluding remarks are then made in the final section.

\section{SOLDER PASTE STENCIL PRINTING}

The solder paste printing process starts by placing a metal stencil over the PCB. Stencil openings (apertures) corresponds to pad location on the PCB where solder paste is required. A moving squeegee is located on top of the stencil to force the solder paste, rolling in front of the squeegee into the stencil openings. When the squeegee has traveled past all stencil openings, the stencil 


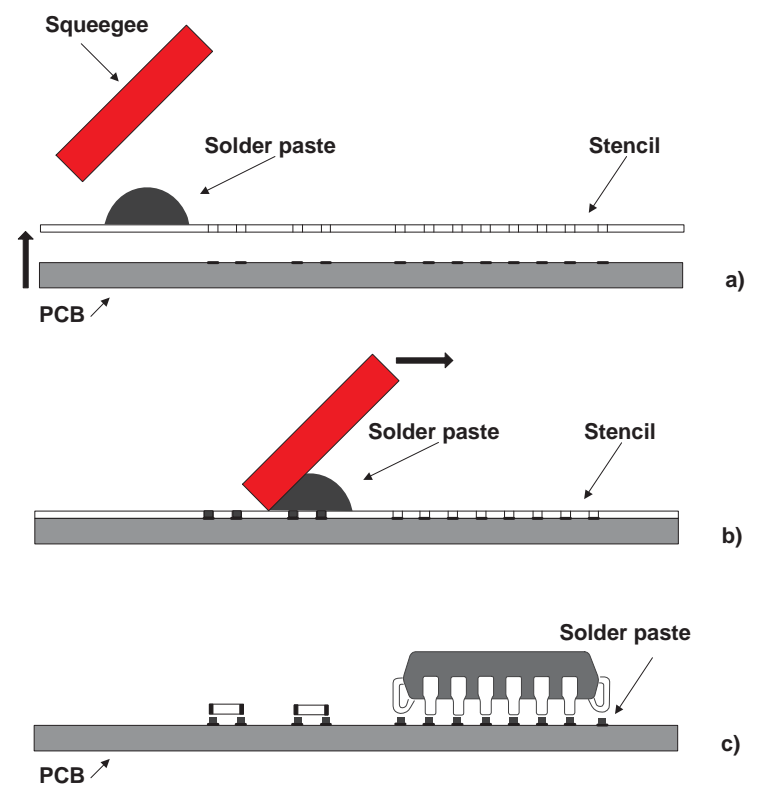

Fig. 3. A schematic of solder paste stencil printing.

is removed and the PCB is ready for component placement. Figure 3 illustrates a schematic diagram of the solder paste stencil printing stage of surface mount PCB assembly.

The height, area and volume of the solder paste deposit is ideally equal to the shape of the stencil apertures. Solder paste deposit for $1.25 \mathrm{~mm} \mathrm{pitch}^{2}$ and higher is generally reliable and close to the ideal shape. The problem arises when fine-pitch elements $(\leq 0.6 \mathrm{~mm}$ pitch $)$ are present on the PCB. For the stencil printing process to deliver the best results, a balance of interactive printer parameters must be achieved. There are many factors which can influence stencil printing performances, either directly or through interaction with other factors. These include squeegee speed, squeegee load, viscosity of paste, enviromental factors etc.

$\overline{2}$ Pitch is the total distance between the centers of two adjacent pads. 
To investigate the quality of each print, the paste deposited on each PCB will be scanned using a fast laser scanner. Producing a digital image of the paste deposit. In the following sections, a short introduction to digital images and the conversion of each image to a set of fuzzy features is given.

\section{FEATURES EXTRACTION}

\subsection{DIGITAL IMAGES}

A digital image, $I(x, y)$, is characterized by a $N_{x} \times N_{y}$ matrix of grey levels. The elements of this matrix are in certain bounds i.e. $I(x, y) \in\left\{G_{l}, G_{l}+1, \ldots, G_{h}\right\}$ where $G_{l}$ and $G_{h}$ are the lowest and highest grey values of pixels respectively.

To enhance the contrast of the digital image so that all grey-levels of grey-scale are utilized, contrast stretching [13] is performed by first shifting all the values so that the lowest grey value begins at 0 , i.e. add to every pixel the difference between the new low value, 0 , and the initial lowest value, $G_{l}$. Next, all pixels are scaled by reassigning all the values by a factor $255 /\left(G_{h}-G_{l}\right)$. Thus, an image $I$ is stretched to the full range of grey-levels i.e. $I_{c s}(x, y) \in 0,1,2, \ldots, 255$. the above expression can be formulated as follows:

$$
I_{c s}(x, y)=\frac{255\left(I(x, y)-G_{l}\right)}{G_{h}-G_{l}}
$$


Let $\mu(I(x, y))$ denote the mapping of all pixels from digital image $I(x, y)$ into $\left[\begin{array}{ll}0 & 1\end{array}\right]$. For any pixel $(x, y) \in I(x, y)$, the grey level of pixel scaled to the range $[01]$ i.e. $\mu(I(x, y))$ is regarded as the membership of pixel $(x, y)$ in the fuzzy set of bright pixels $[8,11]$. In this paper the mapping $\mu(I(x, y))$ is defined by rescaling the contrast stretched image, $I_{c s}(x, y)$, into the range [0 1$]$. It can easily be produced by dividing all $I_{c s}(x, y)$ entries by 255 .

$$
\mu(I)=\frac{I_{c s}}{255}=\frac{I(x, y)-G_{l}}{G_{h}-G_{l}}
$$

Various geometrical properties of $\mu(I(x, y))$ are defined by Rosenfeld [12]. These features are employed extensively for pattern recognition. For instance in [8] the fuzzy geometrical features, as well as textural features are used for fingerprint classification. In our study, the eight fuzzy geometrical features used in $[8,10,12]$ are employed. The features and their definitions are described below.

- Area: The area, $a(\mu)$, of a fuzzy subset of $\mu(I(x, y))$ is defined as follows:

$$
a(\mu)=\sum_{x, y} \mu(I(x, y))
$$

- Perimeter: For a digital image with a fuzzy subset of $\mu(I(x, y))$ the perimeter is defined by:

$$
p(\mu)=\sum_{y} \sum_{x}|\mu(I(x, y))-\mu(I(x+1, y))|
$$




$$
+\sum_{x} \sum_{y}|\mu(I(x, y))-\mu(I(x, y+1))|
$$

where the membership values of two adjacent pixels are subtracted and the absolute differences are added.

- Compactness: The compactness of a fuzzy subset of $\mu(I(x, y))$ having area $a(\mu)$ and perimeter $p(\mu)$ is defined by:

$$
c(\mu)=\frac{a(\mu)}{p^{2}(\mu)}
$$

- Height and Width: The height, $h(\mu)$, and width, $w(\mu)$, of a fuzzy subset of $\mu(I(x, y))$ are defined by:

$$
h(\mu)=\sum_{y}\left\{\max _{x} \mu(I(x, y))\right\}
$$

and

$$
w(\mu)=\sum_{x}\left\{\max _{y} \mu(I(x, y))\right\}
$$

- Length and Breadth: The length, $l(\mu)$, and breadth, $b(\mu)$, of a fuzzy subset of $\mu(I(x, y))$ are defined by:

$$
l(\mu)=\max _{y}\left\{\sum_{x} \mu(I(x, y))\right\}
$$

and

$$
b(\mu)=\max _{x}\left\{\sum_{y} \mu(I(x, y))\right\}
$$

- Index of Area Convergence: The index of area convergence of a fuzzy subset 
of $\mu(I(x, y))$ is defined as follows:

$$
a c(\mu)=\frac{a(\mu)}{l(\mu) \times b(\mu)}
$$

To classify different images corresponding to disparate types of deposit, each image is represented by its fuzzy features explained above and the classification methods will take place on this vector. Therefore, an image $I$ with its fuzzy values $\mu(I(x, y))$ is represented by the fuzzy geometrical feature vector, $\mathbf{V}_{\mathbf{k}}$.

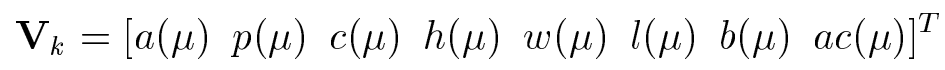

\section{DEFECTS CLASSIFICATION}

Consider a set of fuzzy geometrical features $\bar{V}=\left\{\mathbf{V}_{\mathbf{1}}, \ldots, \mathbf{V}_{\mathbf{k}}, \ldots, \mathbf{V}_{\Gamma}\right\}$, where $\mathbf{V}_{k}=\left[v_{k}^{1}, v_{k}^{2}, \ldots, v_{k}^{m}\right]^{T}$ is a $m$ fuzzy geometrical features vector extracted from the $k$ th image. The fuzzy clustering of $\bar{V}$ into $q$ clusters is a process of assigning a grade of membership to each object $\mathbf{V}_{\mathbf{k}}$ for any cluster $[1,9,16]$. One of the most frequently used clustering algorithms which has been applied is the fuzzy c-means algorithm (FCM) [1]. This algorithm assigns objects, which are described by several features, to different classes with different degrees of membership. An advantage of this method is that it provides an automatic method of forming the membership functions and does not require any initial knowledge about the structure in the feature vectors $[14,17]$. 
The fuzzy clusters can be characterized by a $q \times \Gamma$ class membership function (CMF) matrix $\bar{U}=\left[u_{k}^{\ell}\right]$, whose entries satisfy the following conditions:

$$
\begin{gathered}
\sum_{\ell=1}^{q} u_{k}^{\ell}=1, \quad k=1,2, \ldots, \Gamma \\
0<\sum_{k=1}^{\Gamma} u_{k}^{\ell}<\Gamma, \quad \ell=1,2, \ldots, q
\end{gathered}
$$

where $u_{k}^{\ell}$ is the grade of membership for $\mathbf{V}_{\mathbf{k}}$ object in the $\ell$ th cluster.

In FCM, cluster centers are determined first at the learning stage, and then the classification is made by the comparison of distance between the incoming feature and each cluster center. At the learning stage, cluster centers are obtained by the minimization of a cost function given below:

$$
\begin{aligned}
J(\bar{U}, \bar{C})= & \sum_{k=1}^{\Gamma} \sum_{\ell=1}^{q}\left(u_{k}^{\ell}\right)^{\wp}\left\|\mathbf{V}_{\mathbf{k}}-\mathbf{C}_{\ell}\right\|^{2}, \quad 1 \leq \wp<\infty \\
& \text { and usually } \wp=2
\end{aligned}
$$

where $\bar{C}=\left\{\mathbf{C}_{\mathbf{1}}, \mathbf{C}_{\mathbf{2}}, \ldots ., \mathbf{C}_{\ell}, \ldots, \mathbf{C}_{\mathbf{q}}\right\}$ are $q$ vectors of cluster centers with $\mathbf{C}_{\ell}=$ $\left[c_{\ell}^{1}, c_{\ell}^{2}, \ldots, c_{\ell}^{m}\right]^{T}$ representing $m$ features for the center of the $\ell$ th cluster.

The following algorithm is used [17] to determine the CMFs of each object to a cluster.

(i) Estimate the CMF matrix $\bar{U}$. 
(ii) Calculate cluster centers $\left\{\mathbf{C}_{\mathbf{1}}, \mathbf{C}_{\mathbf{2}}, \ldots, \mathbf{C}_{\mathbf{q}}\right\}$ using the following equation:

$$
\mathbf{C}_{\ell}=\frac{\sum_{k=1}^{\Gamma}\left(u_{k}^{\ell}\right)^{2} \mathbf{V}_{k}}{\sum_{k=1}^{\Gamma}\left(u_{k}^{\Gamma}\right)^{2}} \quad \ell=1,2, \ldots, q
$$

(iii) Update the CMF matrix, $\bar{U}$ to $\bar{U}^{*}$ with:

$$
u_{k}^{\ell}=\frac{1}{\sum_{r=1}^{q}\left(\frac{\left\|\mathbf{V}_{k}-\mathbf{C}_{\ell}\right\|}{\left\|\mathbf{V}_{k}-\mathbf{C}_{r}\right\|}\right)^{2}} \quad k=1,2, \ldots, \Gamma ; \ell=1,2, \ldots, q
$$

(iv) If control error, i.e. $\max _{k, \ell}\left|u_{k}^{\ell *}-u_{k}^{\ell}\right| \leq \epsilon$, stop. Otherwise substitute $\bar{U} \leftarrow$ $\bar{U}^{*}$ and return to step 2.

After a number of iterations, cluster centers satisfy the minimization of cost function, $J(\bar{U}, \bar{C})$, to a local minimum.

\section{SOLDER PASTE STENCIL PRINTING MODELING}

To create a model for the process of printing we propose to map the parameter space $\mathbf{P}_{k}$, to the CMF space $\mathbf{U}_{k}$ explained in the preceding section. The model might be achieved by determining a multi-input multi-output functional relationship of the form:

$$
\mathbf{U}_{k}=f\left(\mathbf{P}_{k}\right)
$$

where $\mathbf{P}_{k}=\left[p_{k}^{1}, p_{k}^{2}, \ldots, p_{k}^{j}, \ldots, p_{k}^{p}\right]^{T}$ and $\mathbf{U}_{k}=\left[u_{k}^{1}, u_{k}^{2}, \ldots, u_{k}^{\ell}, \ldots, u_{k}^{q}\right]^{T}$. This model can be achieved using a number of different modeling approaches. It has been proved that fuzzy rule-based systems (FRBSs) with the structure given in the 
following subsection are universal approximators [15], i.e. they are capable of capturing the nonlinear characteristics of any complex process.

\subsection{FUZZY RULE-BASED SYSTEMS}

The FRBS model we use ( $p$ inputs and $q$ outputs) is in the following form.

$R^{i}$ : If $p_{k}^{1}$ is $\left(\tilde{A}_{i}^{1}\right)_{k}$ and $\ldots p_{k}^{j}$ is $\left(\tilde{A}_{i}^{j}\right)_{k} \ldots$ and $p_{k}^{p}$ is $\left(\tilde{A}_{i}^{p}\right)_{k}$, then $\hat{u}_{k}^{1}$ is $\left(B_{i}^{1}\right)_{k}$ and $\ldots$ $\hat{u}_{k}^{\ell}$ is $\left(B_{i}^{\ell}\right)_{k} \ldots$ and $\hat{u}_{k}^{q}$ is $\left(B_{i}^{q}\right)_{k}$

where $R^{i}$ is the label of $i$ th rule, $p_{k}^{j}: j=1,2, \ldots, p$ is the $j$ th parameter and $\hat{u}_{k}^{\ell}: \ell=1,2, \ldots, q$ is the $\ell$ th CMF values of defects. $\left(\tilde{A}_{i}^{j}\right)_{k}(i=1,2, \ldots, n$ and $j=1,2, \ldots, p)$ are membership functions (MFs) of fuzzy labels assigned to each input parameter, and $\left(B_{i}^{\ell}\right)_{k} \in\left[\begin{array}{ll}0 & 1\end{array}\right]$ are real numbers representing the consequent parameters of FRBS. $n, p$ and $q$ are the numbers of rules, input parameters and number of defect classes respectively. We assume that the universe of variable factors is limited to a lower limit and upper limit bounds, i.e. $p_{k}^{j} \in\left[L L_{j}, \quad U L_{j}\right], \quad j=1, \ldots, p$.

Different shapes of MF for fuzzy values, $\tilde{A}_{i}^{j}$, can be employed e.g. triangular or Gaussian. We further assume that the MFs for each input are normal, i.e.

$$
\sup _{p_{k}^{j}}\left(\tilde{A}_{i}^{j}\right)_{k}=1, p_{k}^{j} \in\left[L L_{j} U L_{j}\right]
$$


The $\ell$ th output of the model, $\hat{u}_{k}^{\ell}$, at the $k$ th print, as a function of printer parameter $p_{k}^{j}: j=1,2, \ldots, p$, is given in the following equation [15]:

$$
\hat{u}_{k}^{\ell}=\frac{\sum_{i=1}^{n} w^{i}\left(B_{i}^{\ell}\right)_{k}}{\sum_{i=1}^{n} w^{i}}
$$

where $w^{i}$ is the rule firing strength given by:

$$
w^{i}=\prod_{j=1}^{p} \mu_{\tilde{A}_{i}^{j}}\left(p_{k}^{j}\right) \quad i=1,2, \ldots, n .
$$

To determine how effectively and accurately the model can estimate the outputs, the following cost function must be optimized.

$$
J_{k}^{\text {model }}\left(\left(\tilde{A}_{i}^{j}\right)_{k},\left(B_{i}^{\ell}\right)_{k}\right)=\min _{\left(\tilde{A}_{i}^{j}\right)_{k},\left(B_{i}^{\ell}\right)_{k}}\left\|\mathbf{e}_{k}\left(\tilde{A}_{i}^{j}, B_{i}^{\ell}, k\right)\right\|^{2}
$$

where $\mathbf{e}_{k}=\mathbf{U}_{k}-\hat{\mathbf{U}}_{k}$. The output of the model, $\hat{\mathbf{U}}_{\mathbf{k}}$ is an estimation of the CMF for the $k$ th print.

To minimize the above optimisation index, the parameters of the FRBS, i.e. $\left(\tilde{A}_{i}^{j}\right)_{k}$ and $\left(B_{i}^{\ell}\right)_{k}$ must be tuned as new data is available. There are many reported methods for adaptive FRBS [5-7] and we have employed the method reported in [7] where a steepest descent gradient method is used.

\section{PRINT QUALITY IMPROVEMENT}

The overall closed loop control systems must be designed in such a way that the sensitivity of the print quality to the process parameters is minimized and 
produces a paste deposit with higher CMF in the class of ideal deposits [2]. The controller task is to produce a set of new parameters $\mathbf{P}_{k+1}$ for the next print based on the data collected from experiment 1 to $k$. To produce a control action the following cost function must be optimized.

$$
J_{k}^{\text {control }}\left(\mathbf{P}_{k}\right)=\min _{\mathbf{P}_{k}}\left\|\mathbf{U}_{k}-\mathbf{U}_{k}^{\mathbf{d}}\right\|^{2}
$$

where $\mathbf{U}_{\mathbf{k}}$ is the CMF vector and $\mathbf{U}^{\mathbf{d}}{ }_{k}$ is the desired CMF for the $k$ th print. $\mathbf{U}^{\mathbf{d}}{ }_{k}$ is a binary vector with all elements equal to zero except one entry corresponding to the class of an ideal deposit which is equal to one i.e. we want to have a print with CMF of one for the ideal shape.

In our investigation we found that the classes of defects can be limited to three major classes of defects in addition to a class of ideal deposit. Therefore, the fuzzy model of the printing process has $q=4$ outputs.

Considering only four classes for the printing results (3 types of defects and one ideal class), the cost function (22) can be rewritten as follows:

$$
J_{k}^{\text {control }}\left(\mathbf{P}_{k}\right)=\min _{\mathbf{P}_{k}}\left(\left|\left(u_{k}^{1}-0\right)\right|+\left|\left(u_{k}^{2}-0\right)\right|+\left|\left(u_{k}^{3}-0\right)\right|+\left|\left(u_{k}^{4}-1\right)\right|\right)
$$

where the fourth class represents an ideal print. From equation (12) we have:

$$
u_{k}^{1}+u_{k}^{2}+u_{k}^{3}+u_{k}^{4}=1
$$


Hence the cost function (22) can be replaced by a simpler optimisation problem of:

$$
J_{k}^{\text {control }}\left(\mathbf{P}_{k}\right)=\max _{\mathbf{P}_{k}}\left(u_{k}^{4}\right)
$$

since

$$
u_{k}^{1} \geq 0 \quad u_{k}^{2} \geq 0 \quad u_{k}^{3} \geq 0 \quad u_{k}^{4} \geq 0
$$

A Simplex search method [4] is used to maximize the cost function (25) from the starting vector $\mathbf{P}_{\mathbf{k}}$. The optimization procedure results in a new parameter vector $\mathbf{P}_{\mathbf{k}+\mathbf{1}}$ which is the local maximum of the FRBS model for the class of ideal deposits.

\section{EXPERIMENTAL RESULTS}

An experiment with 25 combinations of 3 printer parameters $(p=3)$ was conducted. The selected machine parameters are: squeegee angle, squeegee pressure and squeegee speed. The printer parameters for this experiment cover a wide range, chosen to provide data on the machine performance throughout its operational capability. Figure 4 depicts 25 range images for a single Ball Grid Array (BGA) pad obtained from the experiment . Following accurate measurement of the deposit shape and obtaining the range image data, the next step is to calculate $m=8$ fuzzy features of each paste deposit. Consequently 


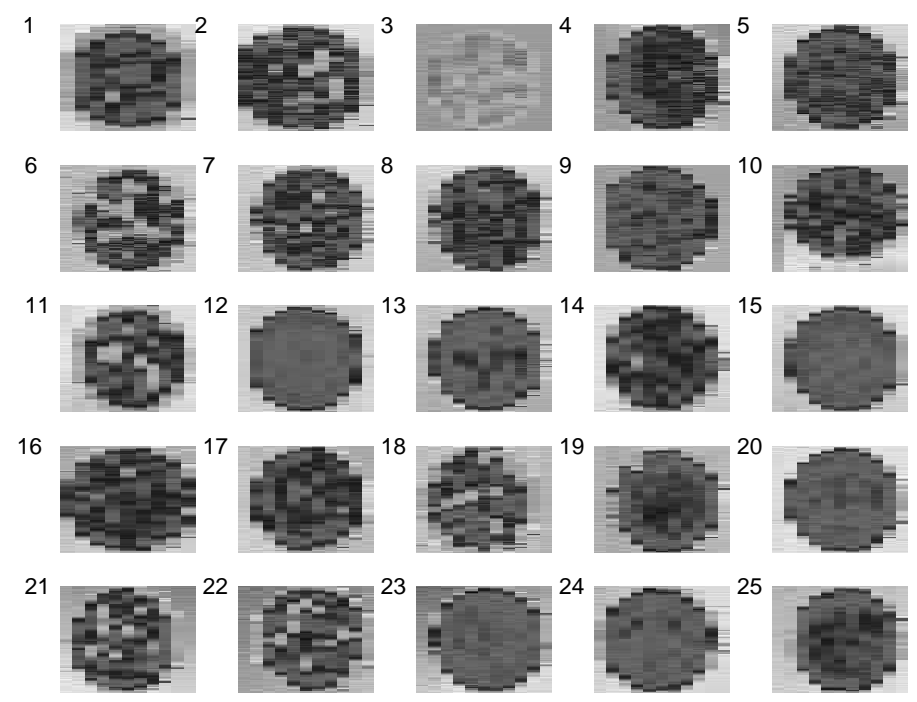

Fig. 4. Images of the paste deposit for a single BGA pad.

we have a matrix $(\bar{V})$ of fuzzy features with 8 columns and 25 rows. Using the matrix of fuzzy features as input data, the fuzzy c-mean classification has been conducted to produce the CMF of each paste deposit for a certain class of defects. For the classification procedure, $q=4$ classes were selected. The CMF values for a single BGA pad are shown in Figure 5. Comparing the results obtained from the CMF grades with the actual images given in Figure 4 shows that the experiment number $9,12,15,20,23$ and 24 are producing the highest CMF for the class of ideal deposits (class 4).

Upon evaluation of the CMF of each image, a fuzzy rule-based model for the ideal class was formed. The original fuzzy rule-based model contains $n=25$ rules with $p=3$ inputs and one output representing the CMF of the ideal class.

After forming the fuzzy model, the parameter values for the next print $\mathbf{P}_{k+1}$ 


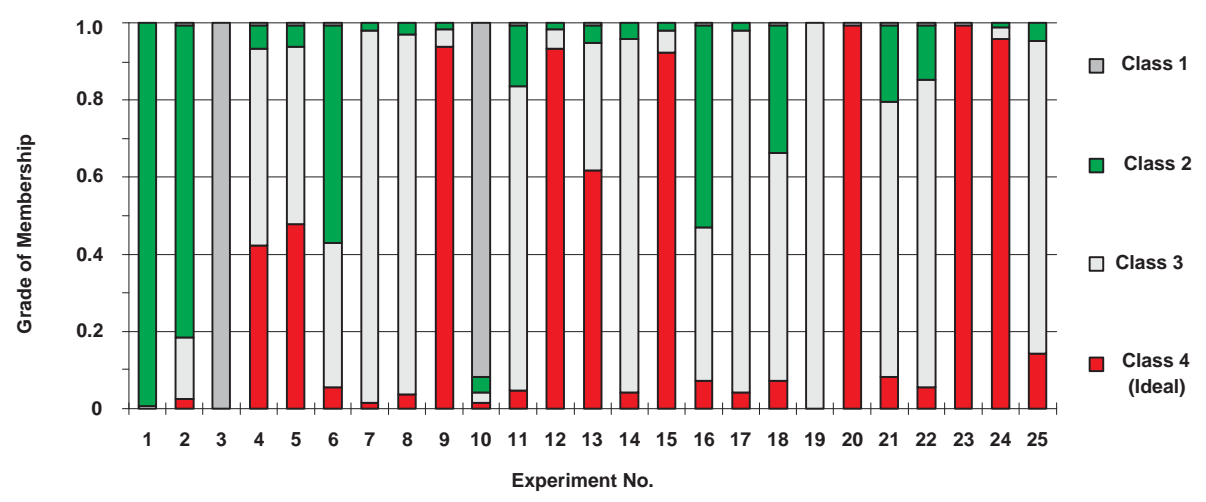

Fig. 5. The CMF values of the 25 print for BGA pads.

are determined by conducting an optimisation technique which detects the maximum effect of each parameter and uses these new values for the next print. The output of the optimisation technique can be either a fuzzy value or a crisp value. The fuzzy value recommends a range of parameters which can provide a good print.

Considering the control action recommended by the controller, a second experiment was conducted concentrating on the ideal region. An experiment with nine combinations of print parameters was conducted to assess the ideal print condition predicted by the controller. The images of the nine paste deposits resulting from the new experiment are illustrated in Figure 6. The new set of experimental data was augmented to the original data and the controller suggested a new set of parameters to improve further the print results. Figure 7 illustrates the results of a confirmation experiment at constant parameter $P_{k}$ settings. The results obtained from the last set of experiment shows that the CMF of new prints are all above 0.8 which is considered as an acceptable print. 


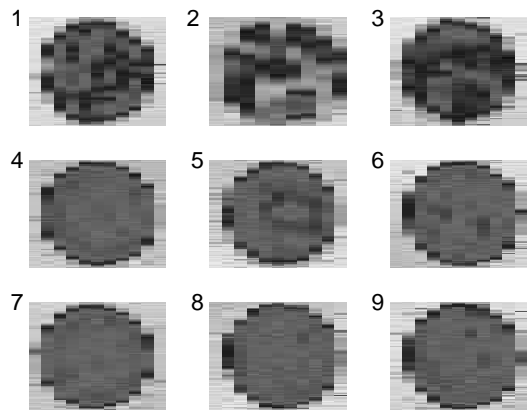

Fig. 6. Images of the paste deposit for the 9 print experiment.
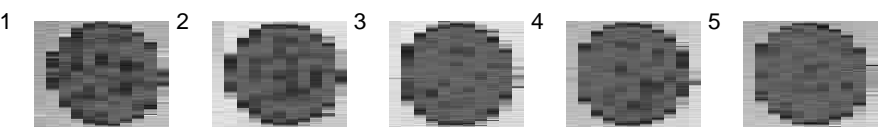

6
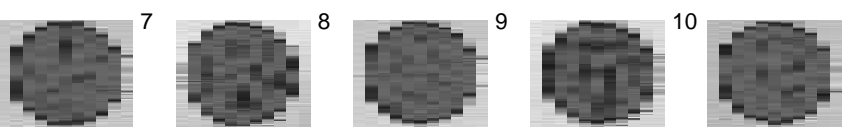

Fig. 7. Images of the paste deposit for confirmation experiment.

\section{CONCLUSIONS}

An algorithm for automatic control of solder paste stencil printing process of SMT is developed to reduce the number of board failures using an adaptive fuzzy controller. The prototype of the proposed automatic control is implemented and it demonstrates the capability of a fuzzy system to model and produce a good control action for solder paste stencil printing. 


\section{References}

[1] J. C. Bezdek, Pattern Recognition with Fuzzy Objective Function Algorithms. New York: Plenum (1981).

[2] C. W. Chung, K. Srihari, J. H. Adriance and G. R. Westby, A "closed loop" system for the realtime control of solder paste stencil printing, Journal of Electronics Manufacturing 5(2):99-109, (1995).

[3] H. Danielson, Surface mount technology with fine pitch components : the manufacturing issues London : Chapman \& Hall (1995).

[4] J. E. Dennis and D. J. Woods, New Computing Environments: Microcomputers in Large-Scale Computing, edited by A. Wouk, SIAM, 1987, pp. 116-122.

[5] J.-S. R. Jang, Neuro-fuzzy modeling and control. Proceedings of the IEEE 38(3):378-405 (1995).

[6] A. Lotfi and A. C. Tsoi, Learning fuzzy inference systems using an adaptive membership function scheme. IEEE Trans. on Systems, Man, and Cybernetics 26(2):326- -331 (1996).

[7] A. Lotfi, H. C. Andersen and A. C. Tsoi, Interpretation Preservation of Adaptive Fuzzy Inference Systems. International Journal of Approximate Reasoning 15(4):379- 394 (1996).

[8] S. K. Pal and S. Mitra, "Noisy fingerprint classification using multilayer perceptron with fuzzy geometrical and textural features", Fuzzy Sets and Systems 80:121-132 (1996). 
[9] S. K. Pal and S. Mitra, "Multilayer perceptron, fuzzy sets, and classification", IEEE Trans. on Neural Networks 3(5):683-697 (1992).

[10] S. K. Pal and A. Ghosh, "Fuzzy geometry in image analysis", Fuzzy Sets and Systems 48:23-40 (1992).

[11] A. Rosenfeld, Fuzzy geometry: an overview, in: Proc. of the First IEEE Int. Conf. on Fuzzy Systems, San Diego, USA, 1992, pp. 113-117.

[12] A. Rosenfeld, "Fuzzy geometry of image subsets", Pattern Recognition Letter 2:311-317 (1984)

[13] D. Vernon, Machine Vision: Automated Visual Inspection and Robot Vision. Prentice Hall, UK (1991).

[14] L. Wang and R. Langari, Complex systems modeling via fuzzy logic, IEEE Trans. on Systems, Man and Cybernetics: Part B 26(1):100-106 (1996).

[15] L. X. Wang and J. M. Mendel, Fuzzy basis functions, universal approximation, and orthogonal least-square learning. IEEE Trans. on Systems, Man, and Cybernetics 22(6):807- 814 (1992).

[16] L. X. Wang, Training of fuzzy logic systems using nearest neighborhood clustering, in: Proc. of the Second IEEE Int. Conf. on Fuzzy Systems, 1993, pp. $13-17$.

[17] Z. Wang, W. Lowrenz, Raj B. K. N. Rao and T. Hope, Feature-filtered fuzzy clustering for condition monitoring of tool wear, Journal of Intelligent Manufacturing 7:13-22 (1996). 